\title{
Luminal cells with an identity crisis
}

Inherited mutations in BRCA1 seem to specifically increase the risk of developing basal-like breast cancers; so, Proia and colleagues investigated how BRCA1 might promote the formation of this subtype of breast cancer.

Using samples from reduction mammoplasty $\left(B R C A 1^{+/+}\right)$and prophylactic mastectomy from individuals with $B R C A 1$ mutations $\left(B R C A 1^{\mathrm{mut} /+}\right)$, Proia and colleagues transformed cell suspensions with four tumorigenic genes (TP53 $3^{\mathrm{R} 175 \mathrm{H}}$, CCND1, PI3KCA and KRAS $\left.S^{\mathrm{G} 12 \mathrm{~V}}\right)$ that are associated with both luminallike and basal-like breast cancer. These cells were introduced into humanized fat pads of mice, and the

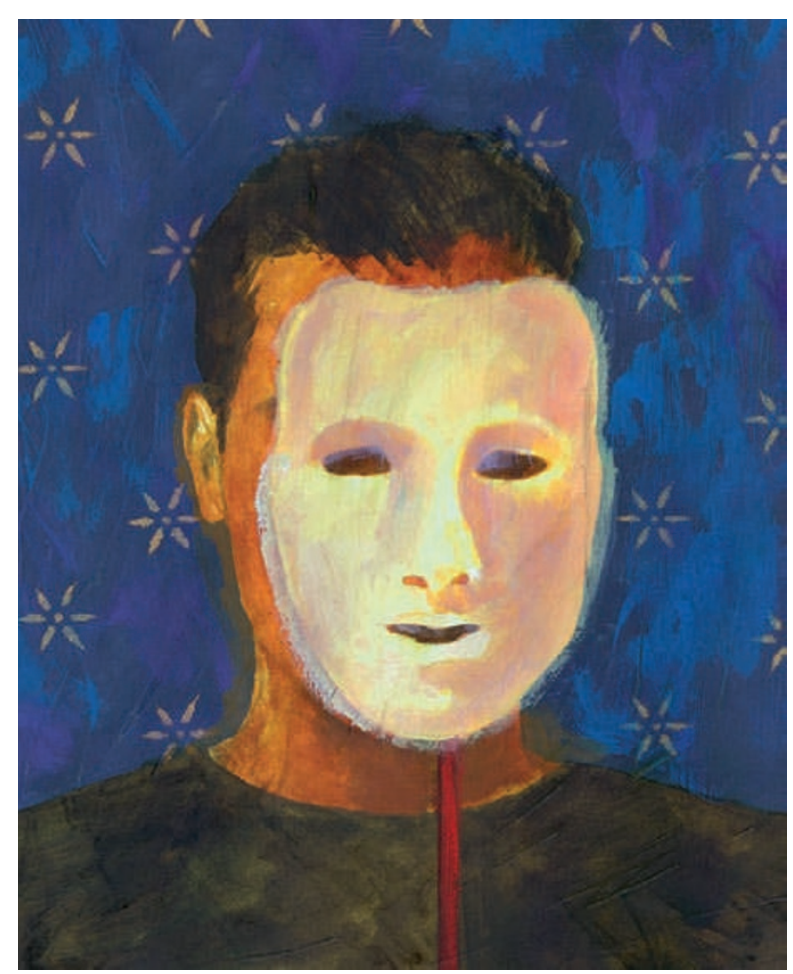

tumours from $B R C A 1^{\mathrm{mut} /+}$ cells had significant upregulation of genes associated with basal and myoepithelial cell differentiation and also had molecular alterations that are characteristic of basal-like breast cancer. To investigate whether $B R C A 1^{\mathrm{mut} /+}$ cells are altered prior to transformation, the authors carried out gene expression profiling of cells from diseasefree individuals; they found that genes involved in chromatin organization and gene expression were differentially expressed in $B R C A 1^{\mathrm{mut} /+}$ cells. In addition, genes associated with luminal differentiation were downregulated, and genes associated with progenitor or myoepithelial cell lineages were upregulated in $B R C A 1^{\mathrm{mut} /+}$ cells. Cell sorting using markers of luminal and myoepithelial differentiation revealed that $B R C A 1^{\text {mut/+ }}$ tissues had significantly increased basal progenitors and decreased luminal progenitors. Indeed, $B R C A 1^{\text {mut/+ }}$ cells injected into humanized fat pads generated immature ductal outgrowths that lacked differentiated luminal features, suggesting that $B R C A 1^{\mathrm{mut} /+}$ luminal progenitors have defects in maturation and differentiation. Next, the authors transformed cells of luminal and myoepithelial lineages from the $B R C A 1^{\mathrm{mut} /+}$ and $B R C A 1^{+/+}$ tissue samples with the four tumorigenic genes and injected them into humanized fat pads; they found that the luminal fraction formed tumours that were comparable to the unsorted transformed $B R C A 1^{+/+}$ and $B R C A 1^{\mathrm{mut} /+}$ cells, whereas the myoepithelial fraction did not. This indicates that, in this model, the cell of origin is in the luminal fraction, probably because these cells fail to undergo luminal differentiation and so retain features of undifferentiated basal and myoepithelial cells.

How is luminal progenitor cell differentiation altered by mutations in BRCA1? The authors reorganized the set of differentially expressed genes between the $B R C A 1^{+/+}$and $B R C A 1^{\mathrm{mut} /+}$ cells according to signalling pathway, and found that the Wnt, Notch and melanogenesis pathways were the most significantly represented in $B R C A 1^{\mathrm{mut} /+}$ cells. As the transcriptional repressor SNAI2 (also known as SLUG) regulates melanocyte development and is a target of the Wnt and Notch pathways the authors examined its expression in the samples from disease-free individuals. In the $B R C A 1^{\mathrm{mut} /+}$ samples, SNAI2 mRNA was unchanged but protein levels were increased. Knock down of SNAI2 in BRCA1 $1^{\mathrm{mut} /+}$ cells reduced the proportion of myoepithelial progenitors and increased the proportion of luminal cells, suggesting that SNAI2 suppresses luminal lineage commitment. Indeed, SNAI2 knockdown increased luminal features and reduced basal-like features of tumour cells from $B R C A 1^{\mathrm{mut} /+}$ tissues and transformed $B R C A 1^{\mathrm{mut} /+}$ cells.

These data clarify a new role for BRCA1 in regulating luminal cell lineage commitment through SNAI2, the expression of which has been associated with basal-like breast cancer.

Gemma K. Alderton

ORIGINAL RESEARCH PAPER Proia, T. A. et al. Genetic predisposition directs breast cancer phenotype by dictating progenitor cell fate. Cell Stem Cell 8, 149-163 (2011) 